\title{
Leukemia in Remission
}

National Cancer Institute

\section{Source}

National Cancer Institute. Leukemia in Remission. NCI Thesaurus. Code C4896.

History of leukemia after therapy with no evidence on the peripheral blood or bone marrow of leukemia (normalization of all hematologic parameters). 